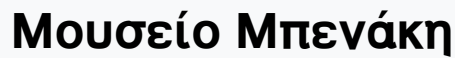

Tóp. 1 (2001)

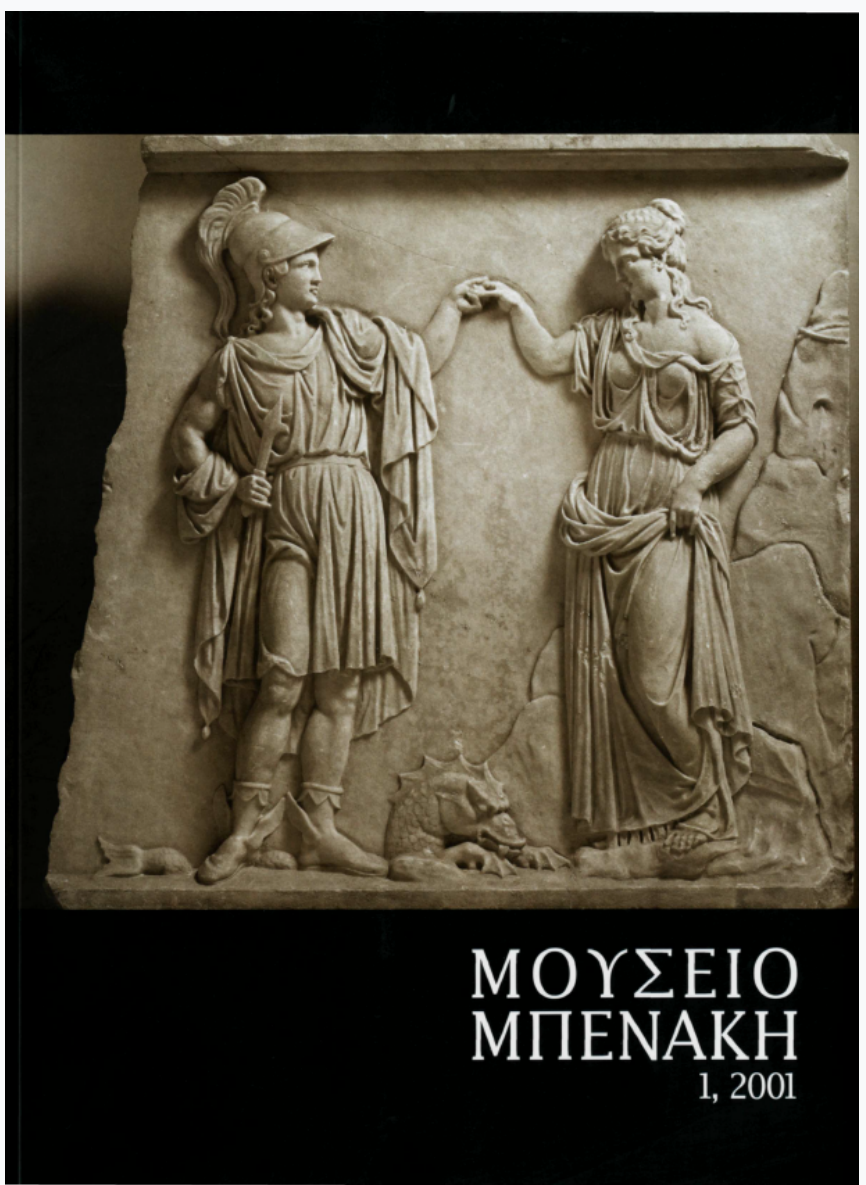

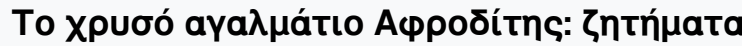
Yvnotótntas

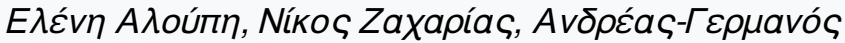
Kapúdas

\author{
doi: $10.12681 /$ benaki.18323
}

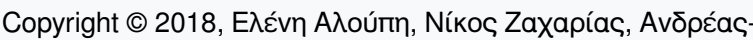

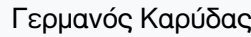

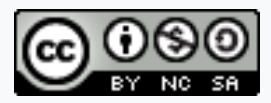

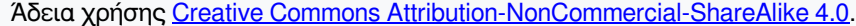

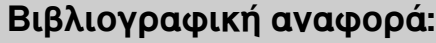

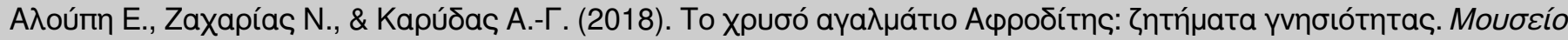
Мпєvákn, 1, 33-40. https://doi.org/10.12681/benaki.18323 


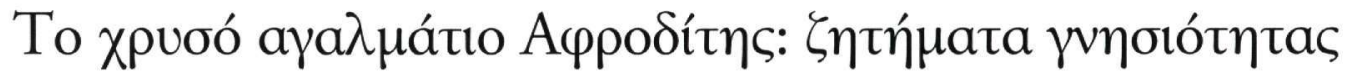

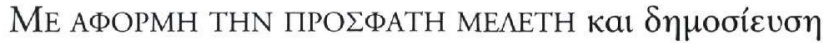

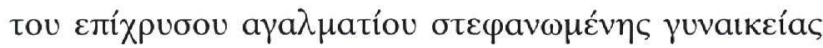

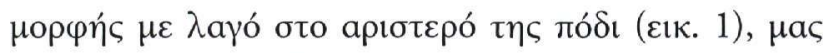

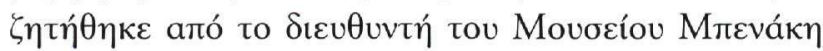

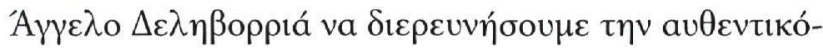

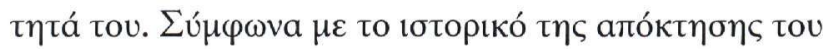

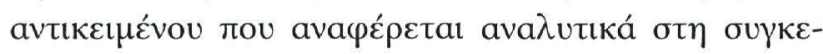

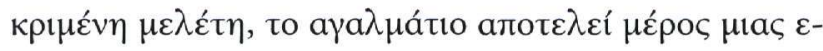

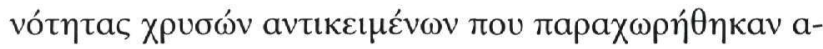

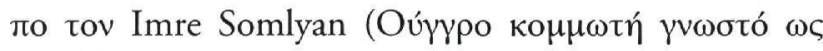

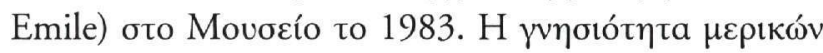

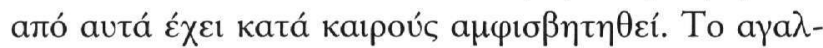

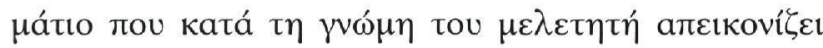

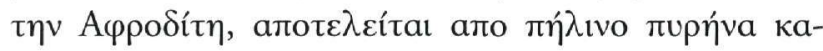
$\lambda \cup \mu \mu \varepsilon ́ v o$ a

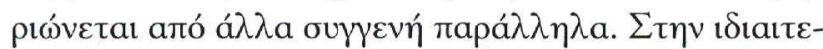

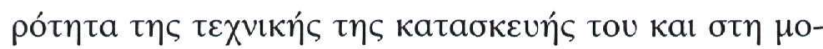

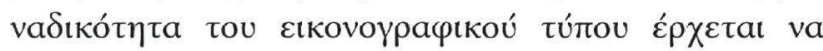

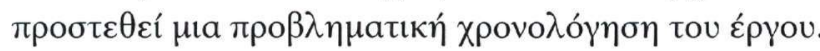

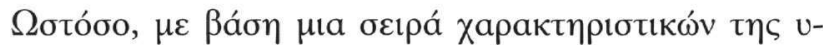

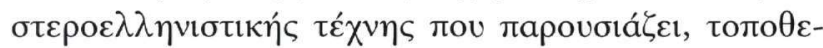

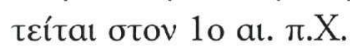

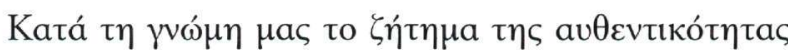

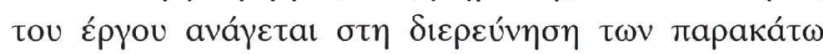
$\tau \rho \iota \dot{v} \pi \varepsilon \rho \iota \tau \tau \omega \sigma \varepsilon \omega v:$

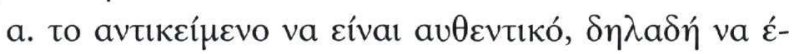

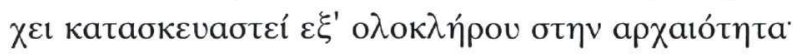

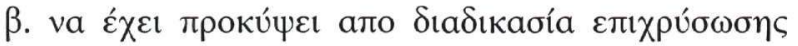

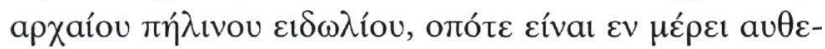

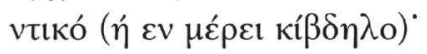

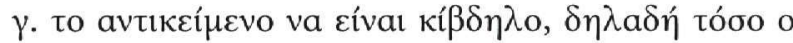

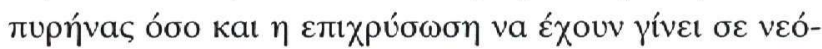

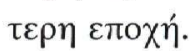

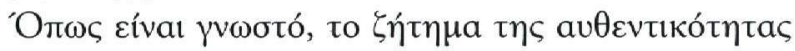

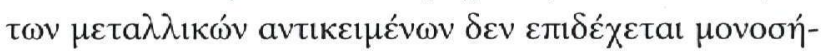

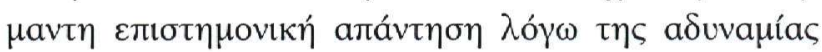

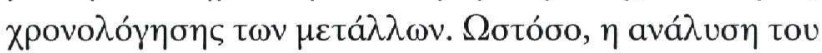

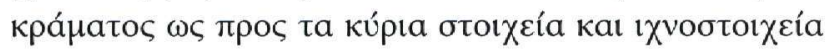

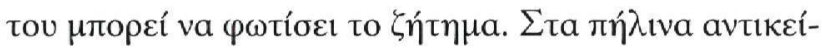

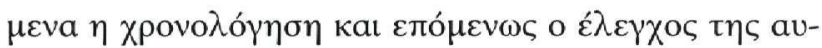

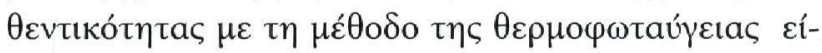

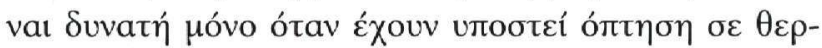
нокрабі́єৎ $\mu \varepsilon \gamma а \lambda \dot{\tau} \varepsilon \rho \varepsilon \varsigma$ ало́ $400^{\circ} \mathrm{C}$.

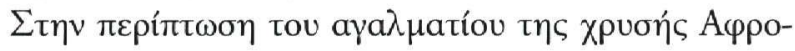

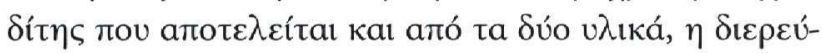

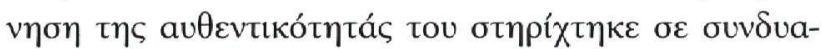

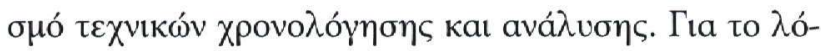

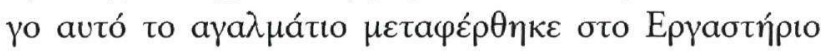

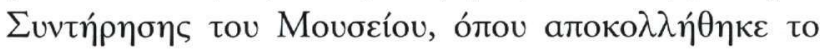

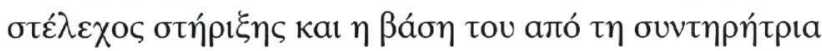

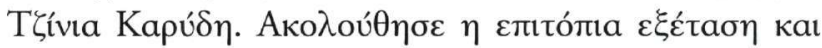

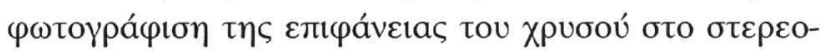

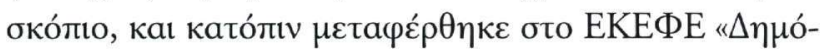

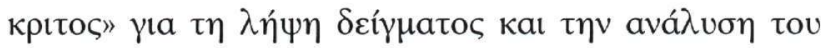

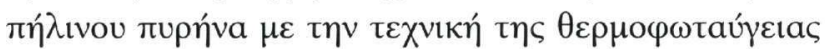

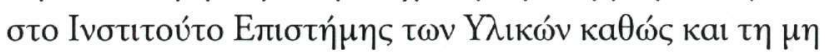

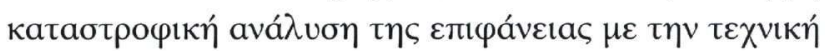

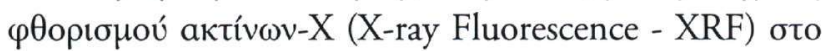

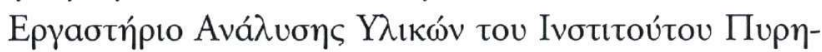
vเки́ৎ Фибкйৎ. 


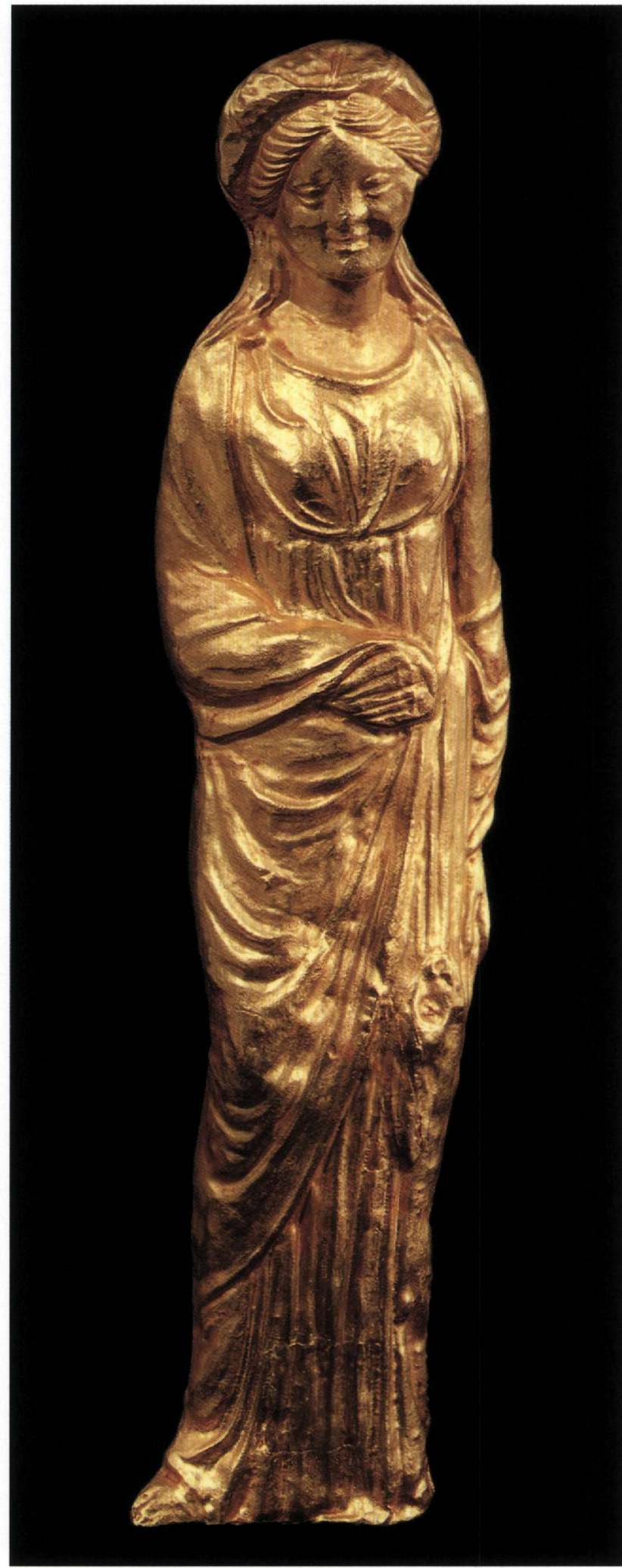

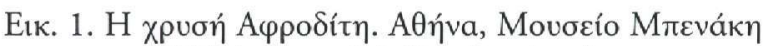
27511 (

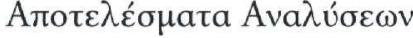

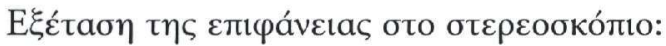

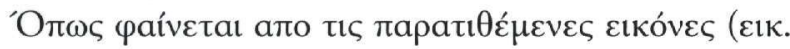

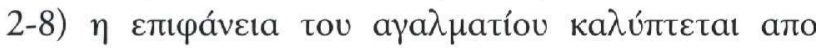

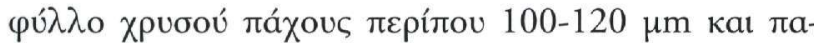

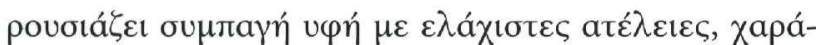

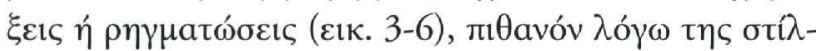

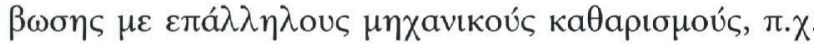

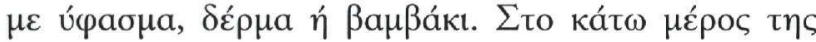

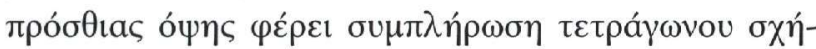

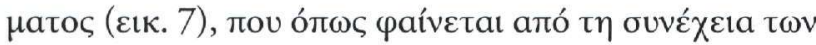

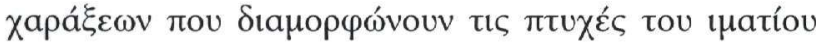

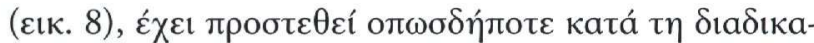
бía катабкєuท́c tou.

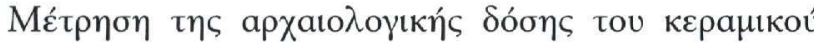

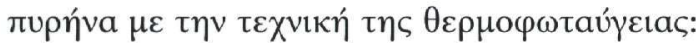

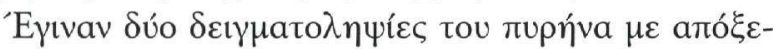

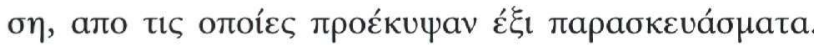

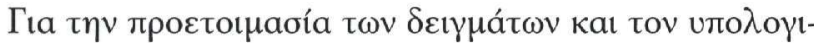

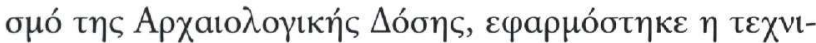

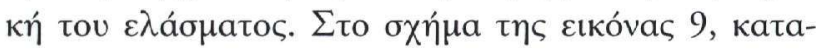

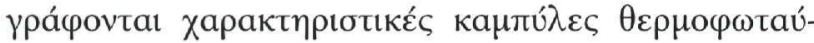

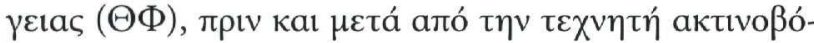

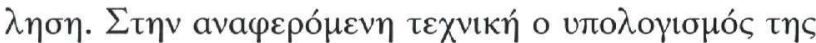

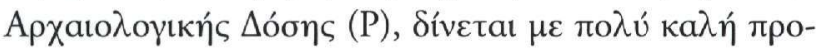

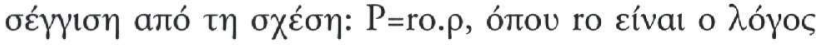

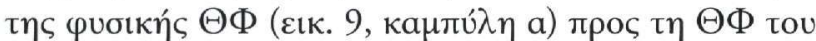

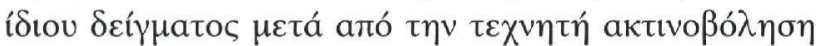

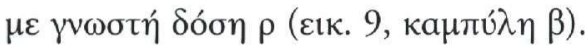

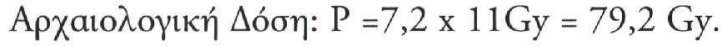

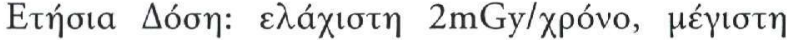
$7 \mathrm{mGy} /$ хрóvo.

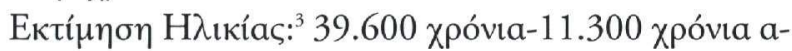

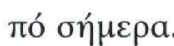

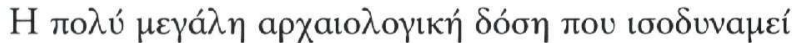

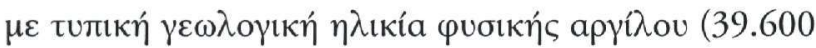

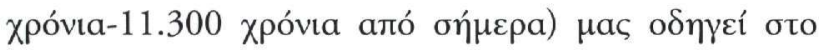

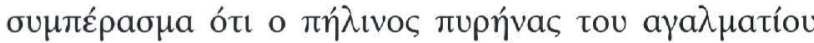

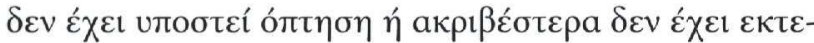

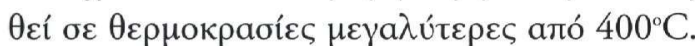

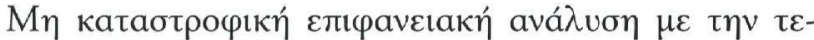

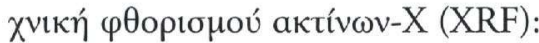

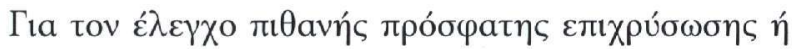




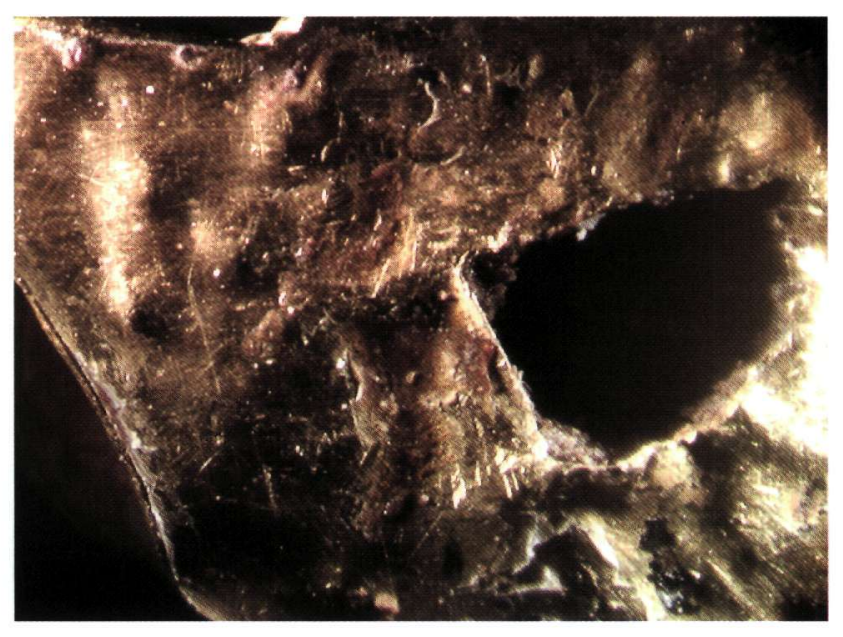

Eıк. 2. Báoๆ $\varepsilon 1 \delta \omega \lambda i ́ o v$

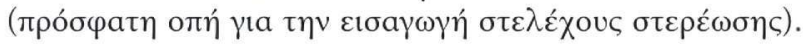

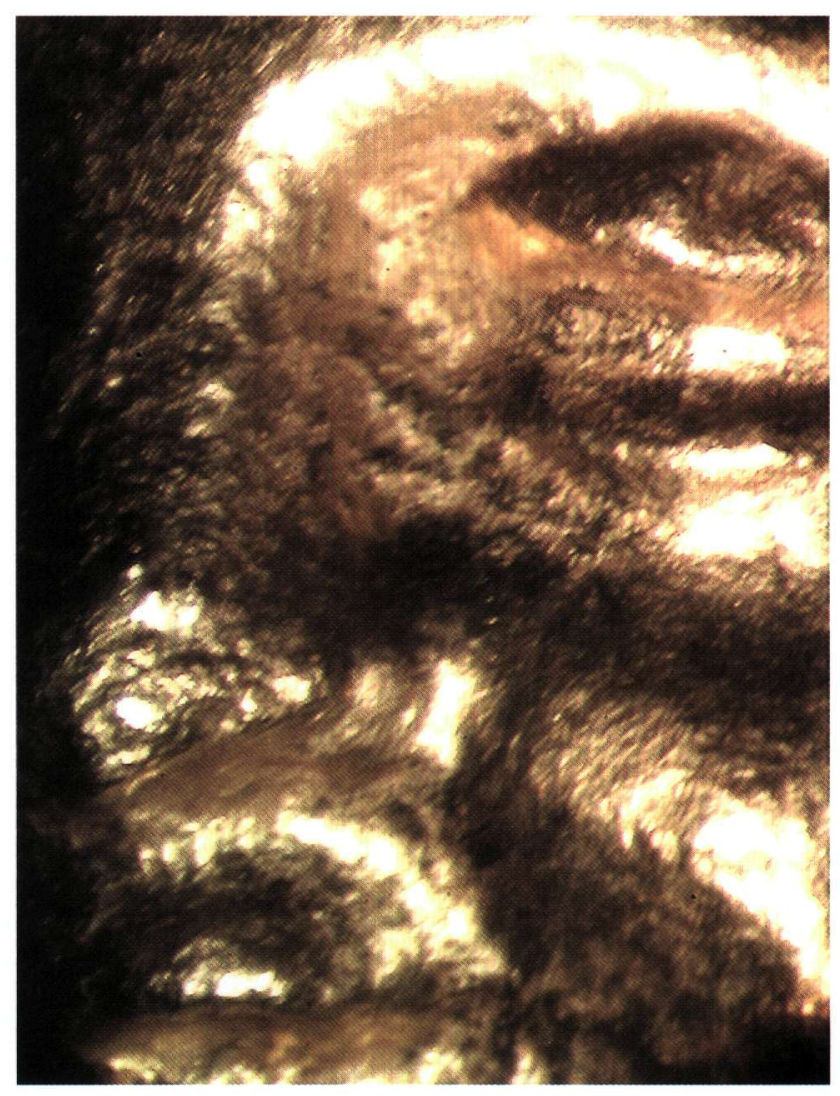

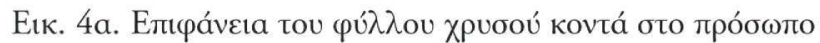

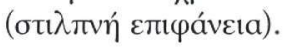

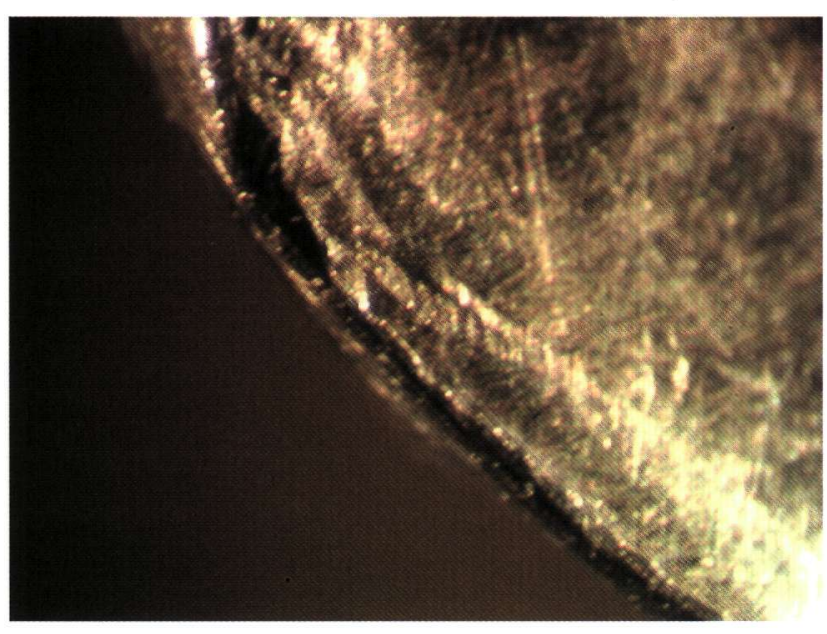

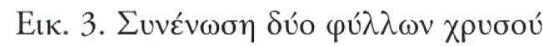

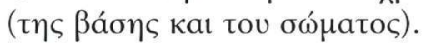

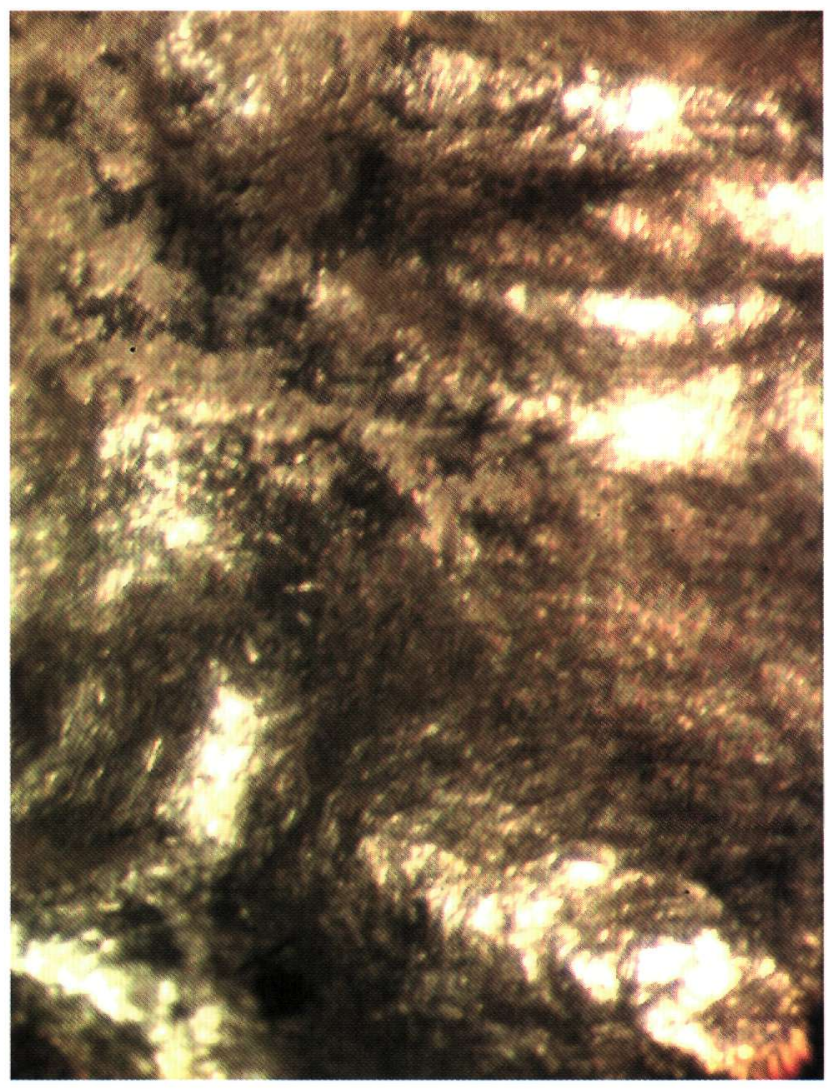

Еıк. $4 \beta . H$ í́ı

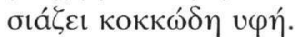




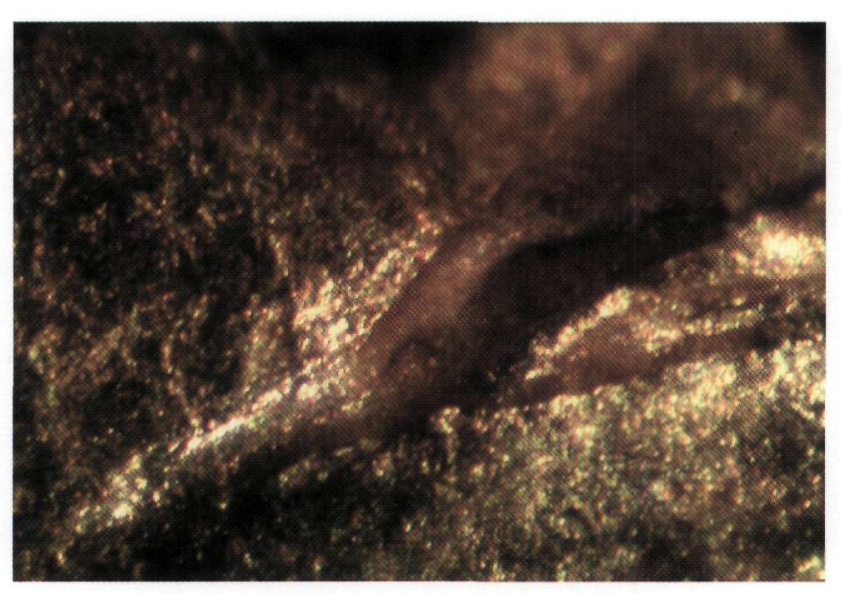

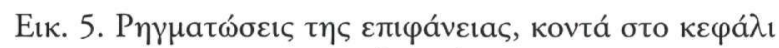
tou גayoú.

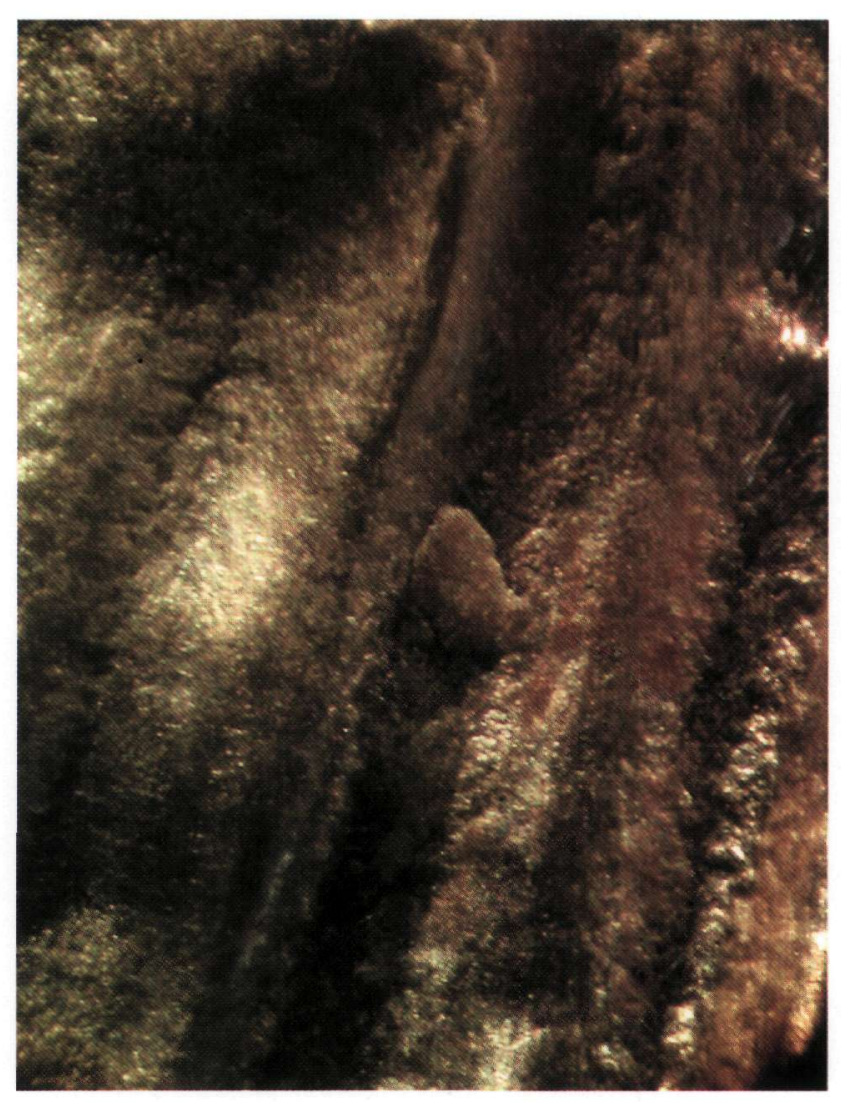

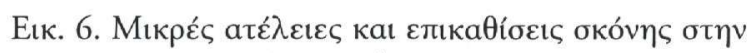

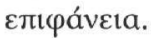

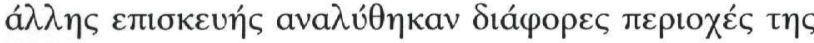

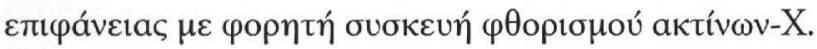
'О

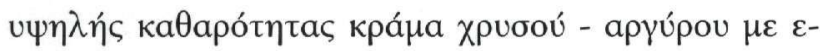

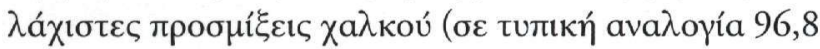

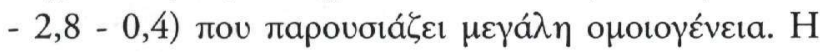

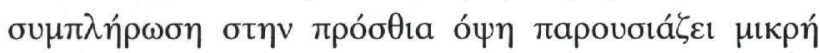

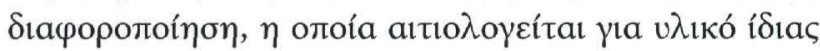

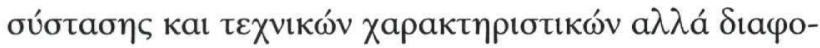

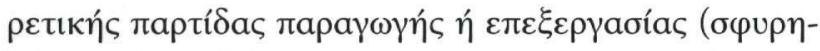

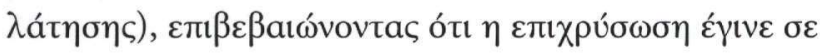

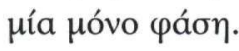

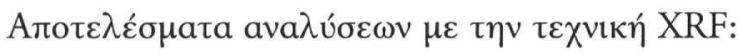

\begin{tabular}{|c|c|c|c|}
\hline Пврьохи́ & $\mathrm{Au}(\%)$ & $\mathrm{Ag}(\%)$ & $\mathrm{Cu}(\%)$ \\
\hline$\pi \lambda a ́ t \eta$ & 96,81 & 2,79 & 0,40 \\
\hline 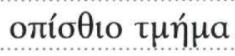 & 96,76 & 2,85 & 0,39 \\
\hline $\begin{array}{l}\pi \rho o ́ \sigma \theta t a \\
\sigma u \mu \pi \lambda \eta ́ p \omega \sigma \eta\end{array}$ & 96,23 & 3,13 & 0,64 \\
\hline 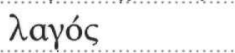 & 96,77 & 2,87 & 0,36 \\
\hline 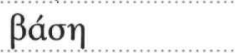 & 97,00 & 2,65 & 0,34 \\
\hline
\end{tabular}

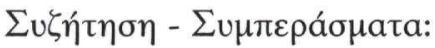

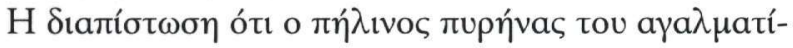

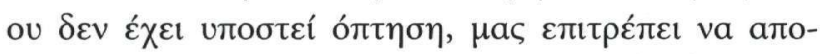

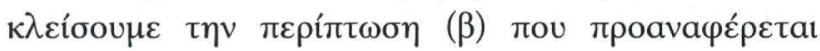

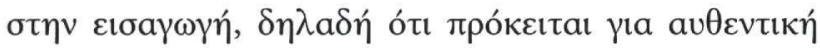

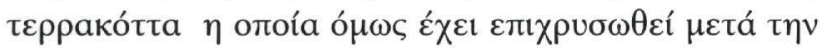

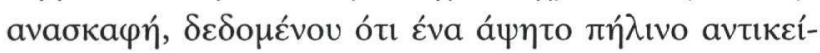

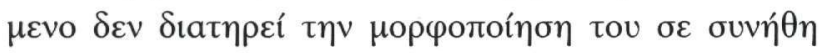

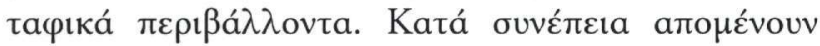

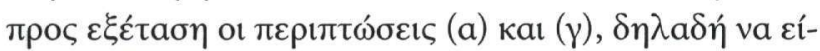

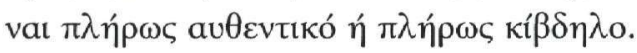

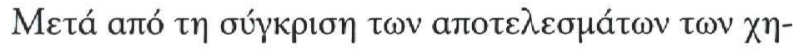

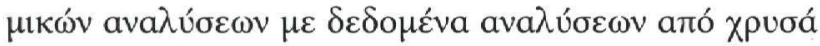

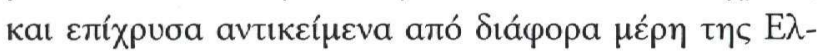

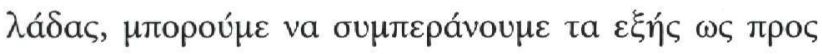

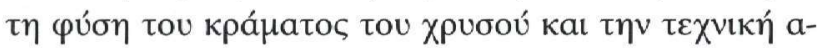

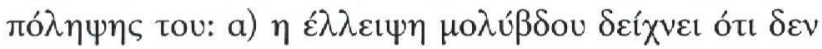

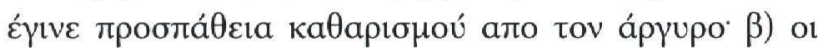

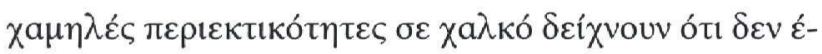

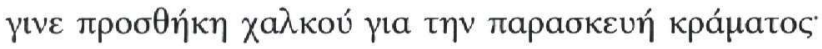

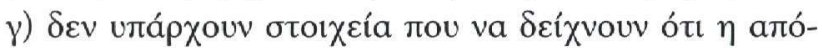

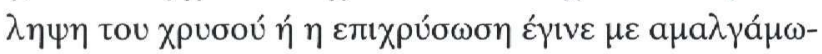

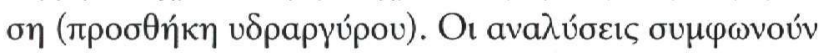




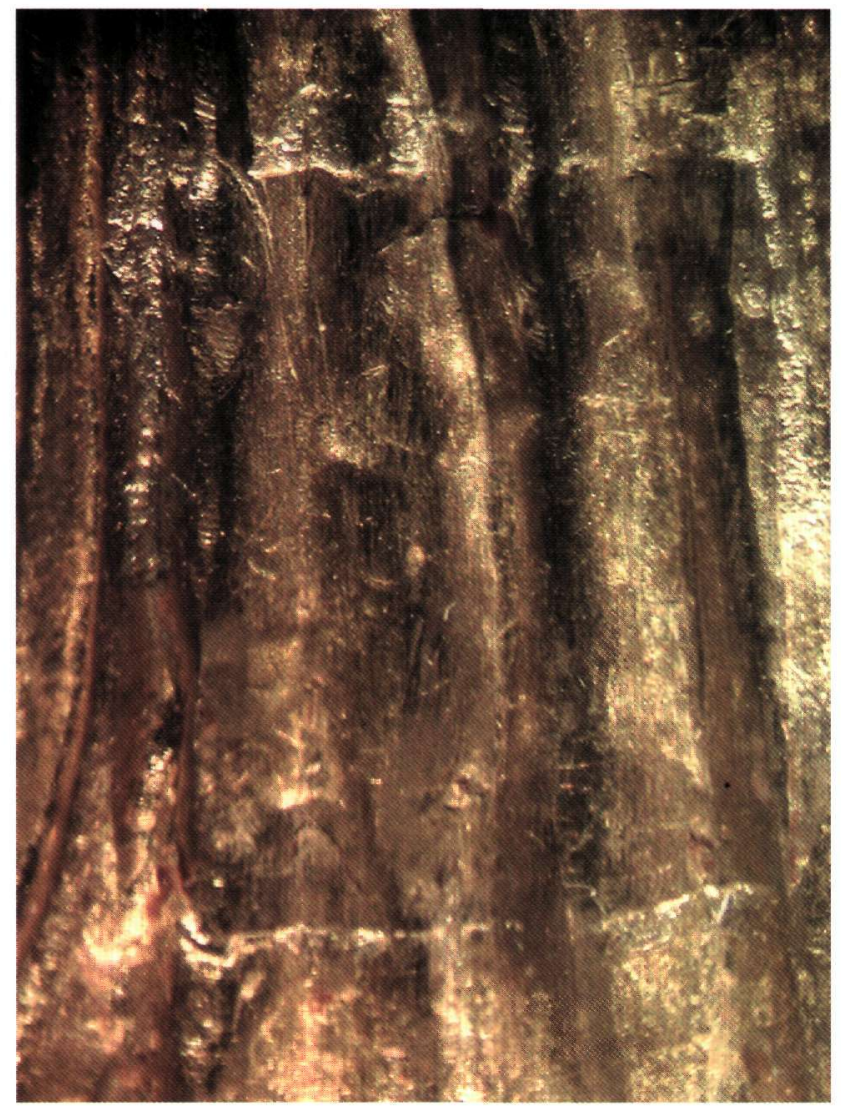

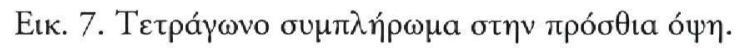

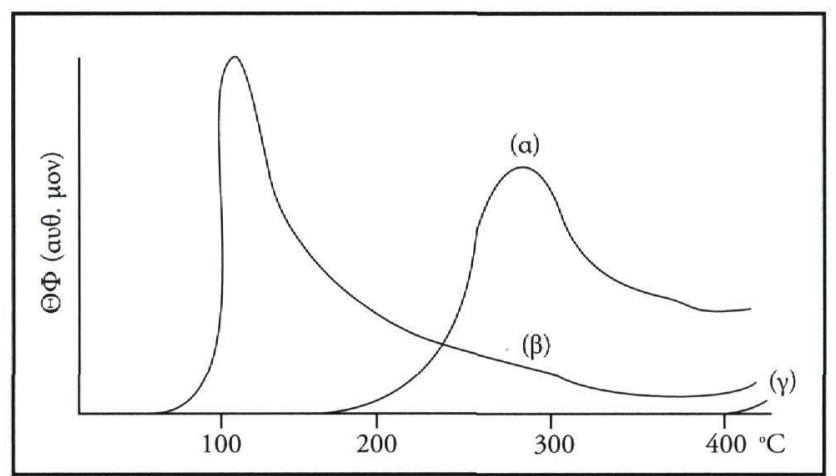

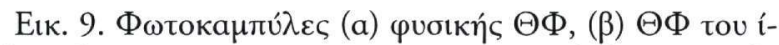

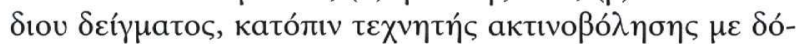

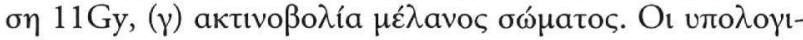

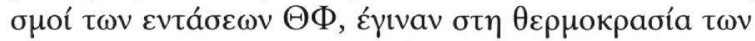
$350^{\circ} \mathrm{C}$.

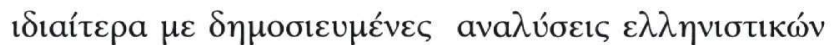

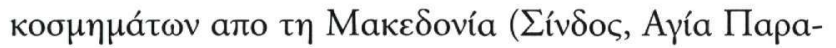

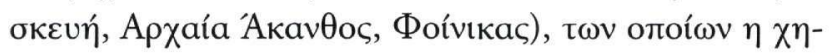

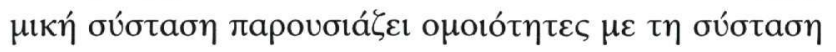

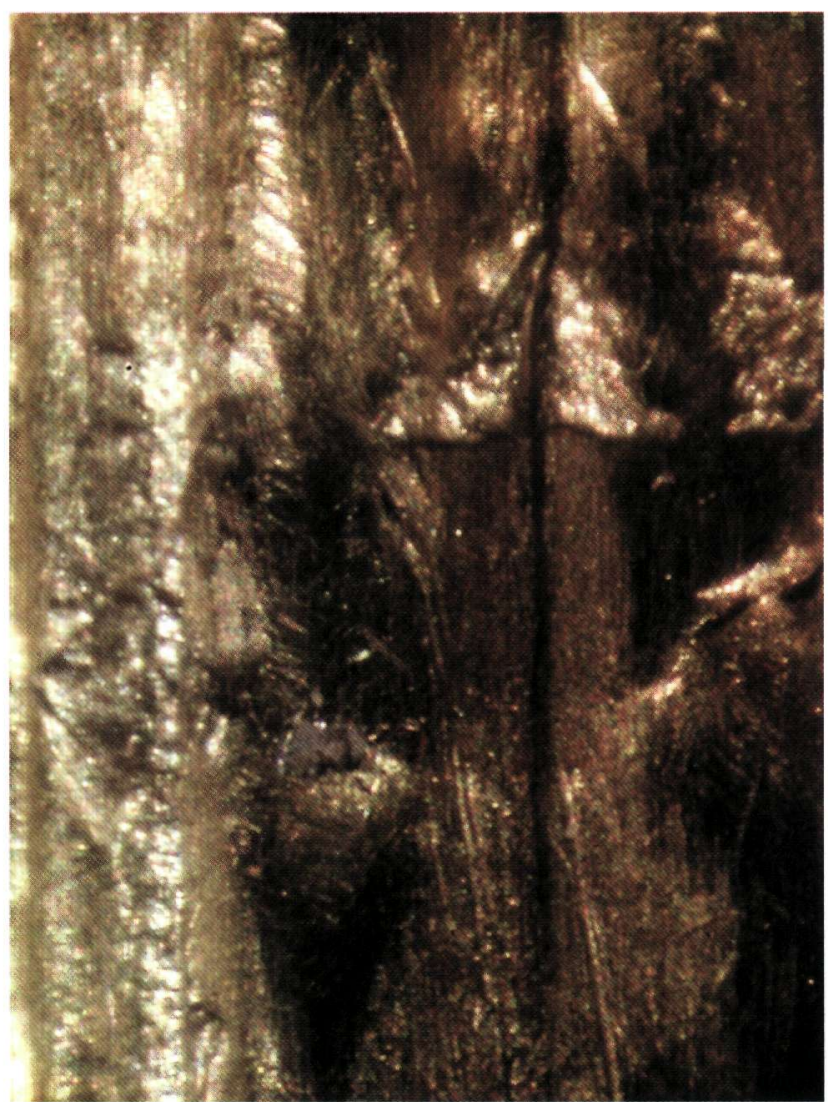

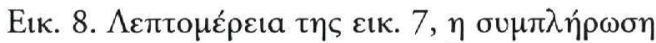

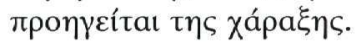

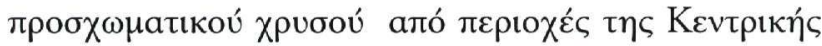

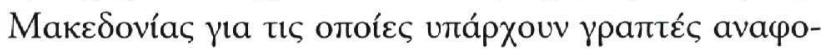


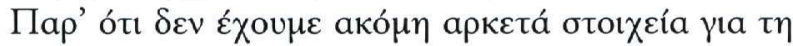

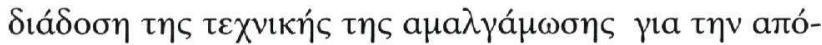

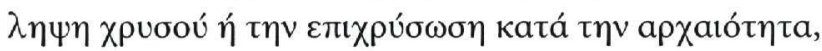

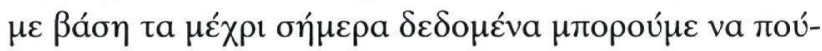

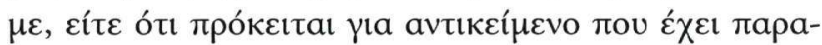

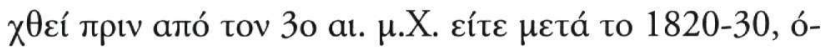

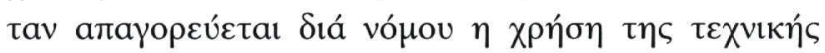

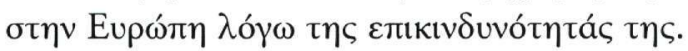

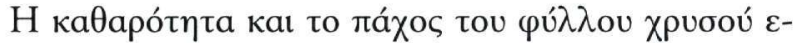

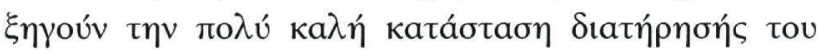

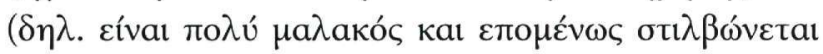

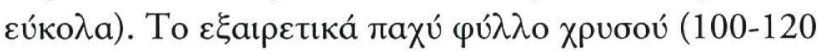

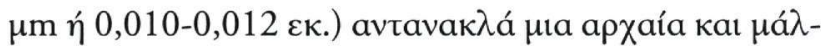

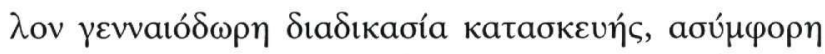

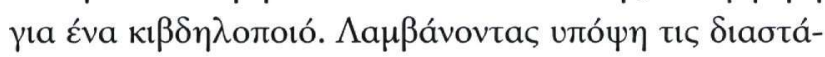

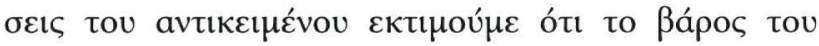




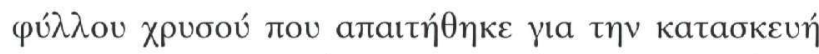

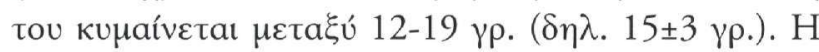

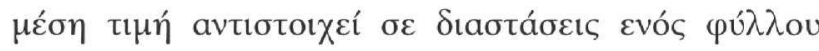

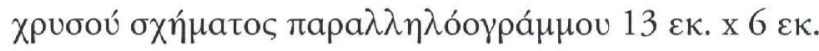

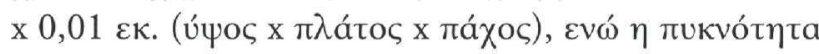

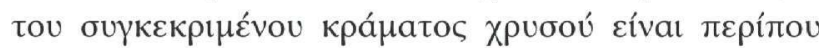

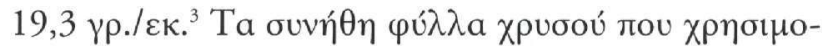

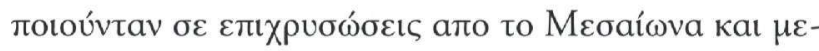

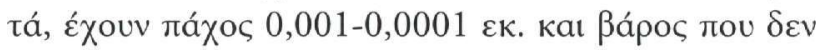

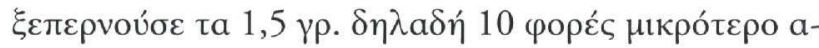

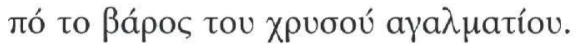

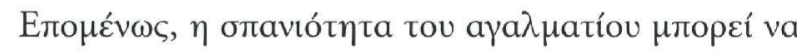
о

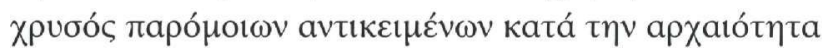

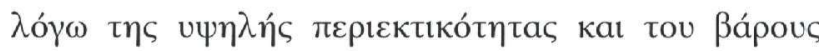

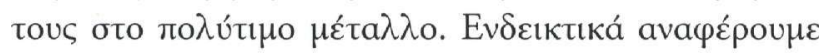

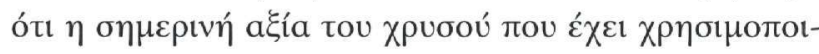

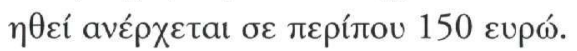

$\sum$ H M EI $\Omega \Sigma$ EI $\Sigma$

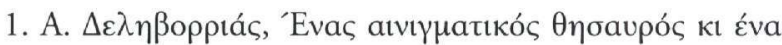

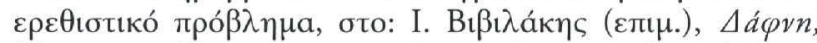

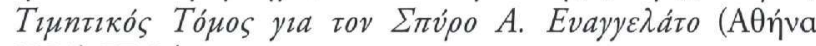
2001) 77-84.

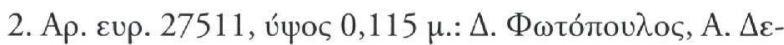

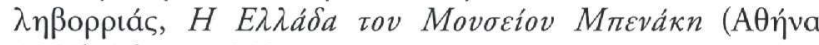
1997) 161 єเк. 283.

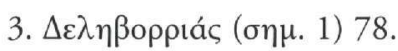

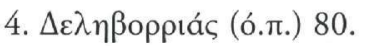

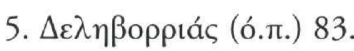

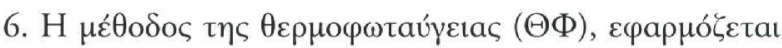

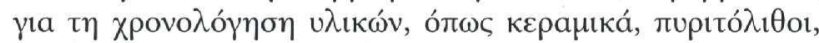

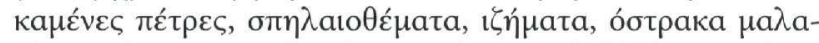

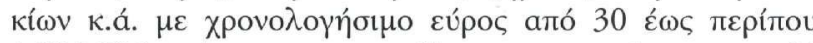

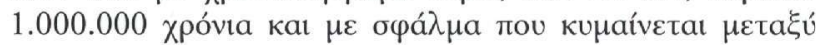

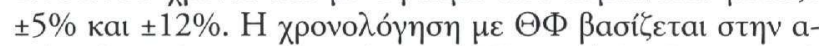

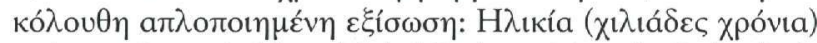
= Apxaı

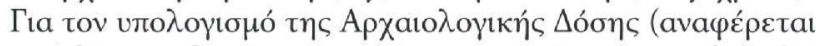

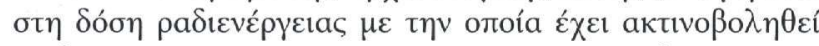

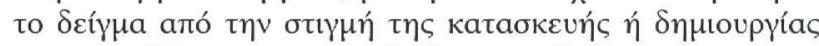

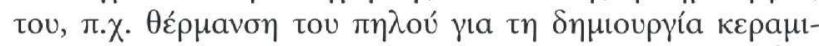

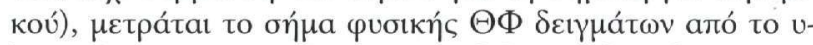

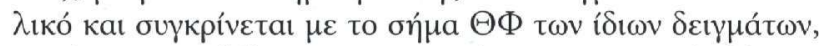

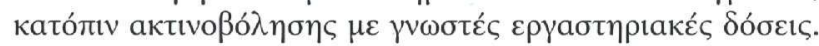

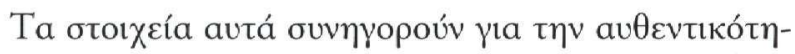

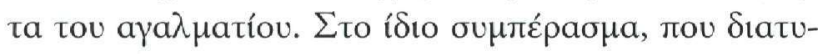

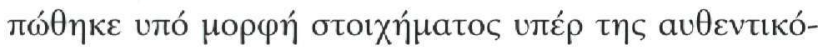

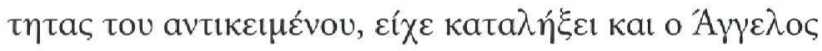

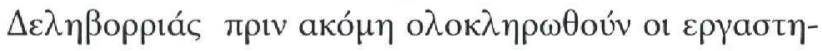

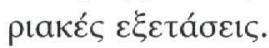

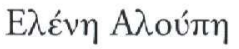

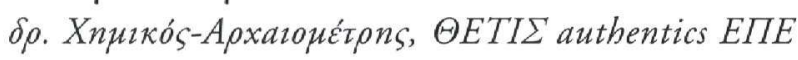

\section{Níkoৎ Zaxapıás}

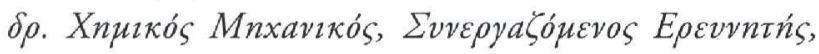

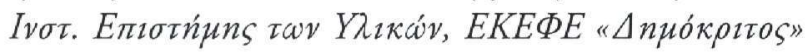

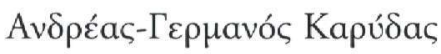

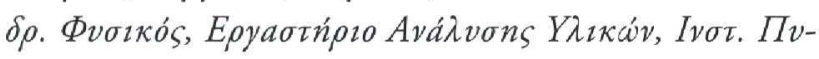

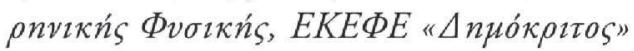

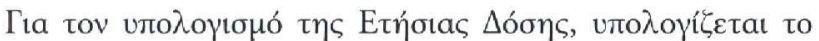

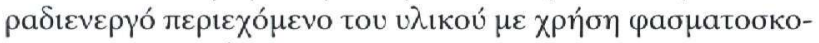

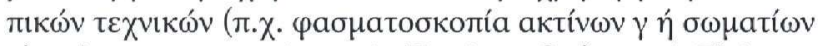

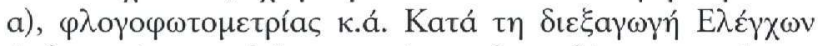

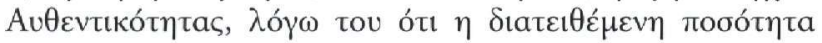

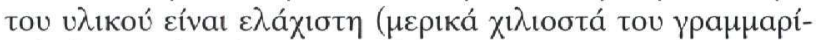

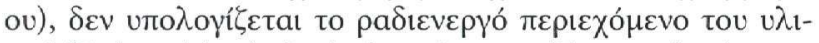

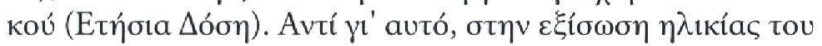

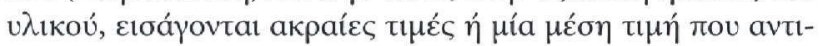

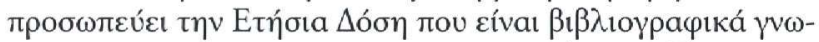

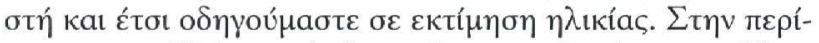

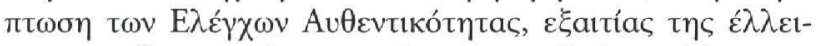

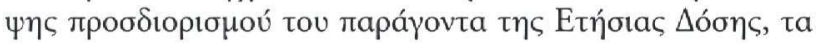

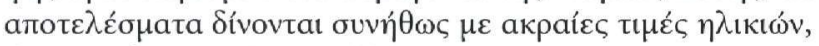

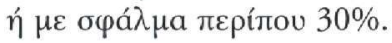

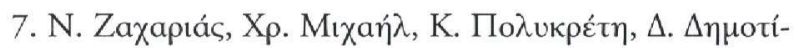

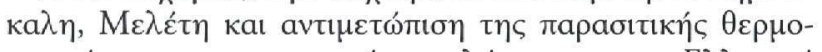

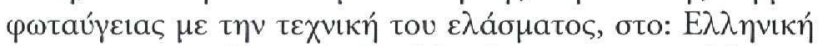

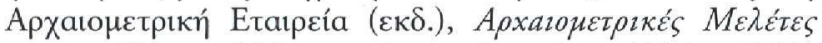

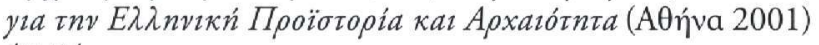
$47-54$.

8. E. Aloupi, A. G. Karydas, T. Paradellis, Pigments of wall paintings and ceramics from Greece and Cyprus. The optimal use of X-ray spectrometry on specific archaeological issues, X-Ray Spectrometry 29 (2000) 18-24.

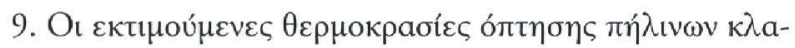




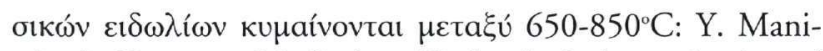
atis, A. Katsanos, M. Caskey, Technological examination of low fired terracotta statues from Ayia Irini Kea, Archaeometry 24 (1983) 191-98.

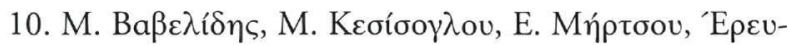

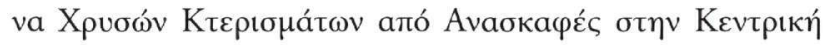

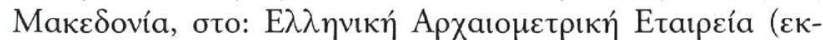

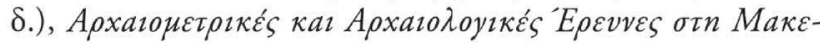

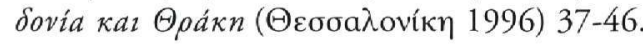

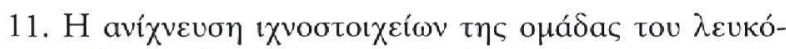

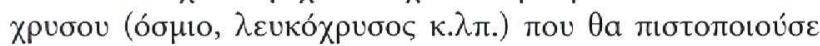

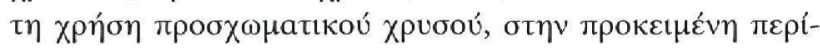

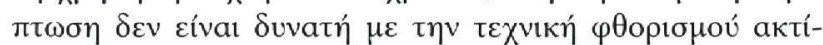

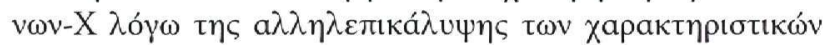

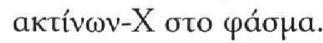

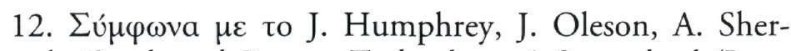
wood, Greek and Roman Technology: A Sourcebook (Lon-

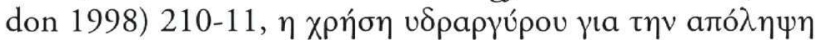

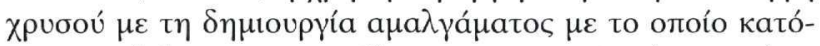

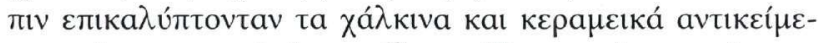

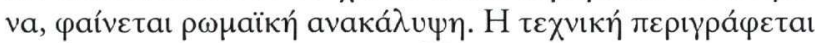

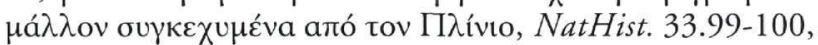

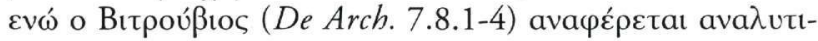

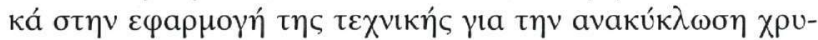

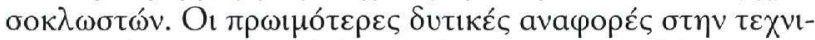

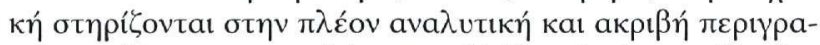

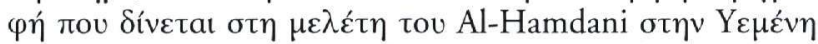
tov $10 \mathrm{o}$ al. $\mu . X$. (P. T. Craddock, Early Metal Mining and Production [Edinburgh 1995] 115-17).

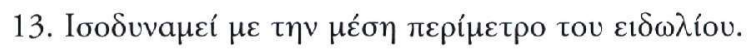

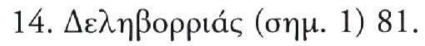

\section{ELENI ALOUPI, NIKOS ZACHARIAS, ANDREAS-GERMANOS KARYDAS}

The gold statuette of Aphrodite: Problems of authentication

The authenticity of a unique and undocumented gold statuette of Aphrodite from the Hellenistic Collection of the Benaki Museum was examined by combining microscopic, analytical and dating techniques. The statuette consists of a clay core covered by a gold layer. The composite nature of the statuette leads to the formulation of the following three hypotheses: (1) both the core and the gold layer are ancient and thus the object is authentic, (2) the gold layer has been applied in modern times on ancient terracotta and (3) both core and gold layer are modern and the object is a complete forgery. Examination under the stereoscope revealed a smooth surface with very few imperfections, scratches or cracks. On the lower front elevation a square gold patch is visible; however the continuity of the relief lines across the patch suggests that the whole surface was covered in a single operation. Moreover the presence of the patch implies that the surface was covered by a gold leaf technique, rather than by following an amalgamation procedure. Where measurable on the base, the thickness of the leaf is about 100 $120 \mu \mathrm{m}$ (i.e. $0.010-0.012 \mathrm{~cm}$ ). The overall smoothness of the surface can be attributed to successive polishing treatments.
Thermoluminescence measurements of the clay core material showed typical geological doses implying that the core has not been fired above $350^{\circ} \mathrm{C}$. Though this precludes the possibility of dating, it provides a very important clue concerning the question of authenticity, in view of the fact that unfired clay objects do not retain their sculptured features following burial. This allows us to exclude the hypothesis that the object is the result of a modern gilding on ancient terracotta.

Non-destructive X-ray fluorescence analysis of the gold surface revealed a high quality gold-silver alloy with a low copper content. Typical percentage by weight is $96.8 \%$ gold, $2.8 \%$ silver, $0.4 \%$ copper. The absence of lead shows that the gold has not been refined by removing silver. Similarly, the absence of mercury suggests that the gold was not obtained by an amalgamation procedure. The composition of the gold alloy broadly agrees with the analyses of gold artefacts found in several Hellenistic Macedonian sites (i.e. Sindos, Ag. Paraskevi, Phoenix). The purity and thickness of the gold layer account for the excellent conservation of the statuette. Furthermore, the thickness of the layer clearly exceeds the minimum required to produce the desired effect. Indeed, from the Middle 
Ages onwards the typical thickness of the gold leaf in gilded objects is of the order of $0.001-0.0001 \mathrm{~cm}$. The total weight of gold in the Aphrodite statuette is of the order $15 \mathrm{gr}$ whereas $1-2$ gr would have sufficed to produce the same effect. This generous use of gold may account for the rarity of similar objects due to the advantages of recycling the precious metal. A converse argument would suggest such an unnecessary waste would not be the practice of a modern forger. The current value of the gold used is about 150 Euro. In conclusion, the combination of all available evidence suggests that the gold Aphrodite in the Benaki Museum is not a forgery and strongly supports the conclusion reached independently by Prof. A. Delivorrias. 